\title{
Der Alterungsprozess - eine chronische Erkrankung mit oft tödlichem Ausgang?
}

Daniela Cerqui

In einer Pressemitteilung vom 6. November $2014^{1}$ sprach die Weltgesundheitsorganisation (WHO) vom „guten Altern“ als einer weltweiten Priorität. Aus dieser Priorität könnte eine Herausforderung für das öffentliche Gesundheitswesen werden. Doch was steckt hinter "gutem Altern“?

\section{Bedeutet gutes Altern nicht zu altern?}

Mit Bezug auf einen Artikel in der britischen medizinischen Fachzeitschrift The Lancet unterstrich der Kommunikationsbeauftragte in der Pressemitteilung, „dass angesichts der Tatsache, dass Menschen überall auf der Welt immer älter werden, der Anstieg chronischer Erkrankungen und die sinkende Lebensqualität gerade dabei sind, zu einer großen Herausforderung für das öffentliche Gesundheitswesen zu werden.“ Ich glaube, dass man darüber hinaus dazu neigt, das Altern selbst oder vielmehr der Alterungsprozess - implizit als chronische Erkrankung mit tödlichem Ausgang für all diejenigen anzusehen, die nicht schon früher durch einen Unfall oder eine andere „Pathologie“ aus dem Leben scheiden.

Hinter „gutem Altern“ steckt also „nicht altern“. Laut Definition der WHO sind chronische Krankheiten Erkrankungen von langer Dauer, die sich in der Regel langsam entwickeln. Während eine akute Erkrankung eine vorübergehende Zäsur darstellt, geht bei einer chronischen Krankheit der frühere Zustand definitiv verloren (Rossi 2009:117), und wie Baszanger unterstreicht, liegt es häufig an dem verfügbaren Wissen, dass der Tod nicht die unmittelbare Perspektive darstellt und die Krankheit damit chronisch geworden ist (1986: 4). Nun muss man sich eingestehen, dass die Zeit nicht alle Wunden heilt - andererseits aber auch feststellen, dass die stetige Erhöhung der Lebenserwartung mit den Wissensfortschritten auf dem Gebiet der Humanbiologie zusammenhängt. Das Altern ist also tatsächlich eine chronische Erkrankung, die zum Tode führt! Es ist jedoch gleichzeitig paradox und eine Ironie, dass das Altern an sich nicht als eine Todesursache angesehen wird. Das Schweizer Bundesamt für Statistik (BFS) ${ }^{2}$ weist darauf hin, dass in der Schweiz die meisten Todesfälle auf eine begrenzte Anzahl an Ursachen zurückzuführen sind: Herz-Kreislauf-Erkrankungen, Krebs, Demenz, Atemwegserkrankungen sowie Unfälle und gewaltsamer Tod. „Alt“ zu sein ist also offiziell kein hinreichender Grund für das Sterben mehr. Dennoch wird in einer Gesellschaft, die es geschafft hat, die Kindersterblichkeit einzudämmen, das Alter mit dem Tod assoziiert.

\footnotetext{
${ }^{1}$ http://www.who.int/mediacentre/news/releases/2014/lancet-ageing-series/fr/

${ }^{2}$ http://www.bfs.admin.ch/bfs/portal/fr/index/themen/14/02/04/key/01.html\#parsys_90762
} 


\section{Verschiebung der Norm}

Der Begriff Normalität hat keinen absoluten Wert (Leblanc 2002 : 149). „Behinderung“ oder „Gesundheit“ sind soziale Konstrukte. Die Frage, ab welchem Alter man ,alt“ wird, steht also zur Diskussion, da all diese Begriffe kulturell bedingt sind und sich mit der Zeit verändern können. Ein Kennzeichen unserer Gesellschaft scheint es zu sein, dass diese ständig neue Behinderungen und Erkrankungen „erfindet“, indem sie u. a. dafür sorgt, dass bestimmte Verhaltensweisen medizinisch behandelt werden. Beispielsweise zeigt Dupanloup (2001) gut auf, dass bei Kindern, die früher lediglich als „wild“ beschrieben worden wären, heutzutage die Diagnose Hyperaktivität gestellt wird und sie daher als krank gelten. Auch die Änderung der Insulinschwelle, ab der jemand als Diabetiker gilt, oder der Höhe des Blutdrucks, ab der jemand als Hypertoniker eingestuft wird, hat zu einer Zunahme der betroffenen Personen geführt und damit Patienten ,geschaffen“, die offiziell als erkrankt gelten. Der von Payer (1992) geprägte Begriff des „disease mongering“ bezieht sich auf die „Erzeugung“ von Krankheiten bzw. die Erfindung neuer Krankheiten oder gesundheitlicher Zustände, die bisher als normal galten und zu Pathologien gemacht werden, um den Arzneimittelmarkt zu beleben. Man kann durchaus noch einen Schritt weiter gehen und behaupten, dass es in diesem Prozess nicht nur um den Umsatz von Pharmaunternehmen geht, die demnach den Rest der Gesellschaft manipulieren, um daran zu verdienen, sondern um das Phänomen einer ganzen Gesellschaft, in der Leistungssteigerung als Ziel für jeden und in allen Bereichen gilt, z. B. in sportlicher, intellektueller oder auch sexueller Hinsicht. Der „ideale Bürger“ ist ein gesunder und zunehmend auch junger Weißer, und wenn man vom „besten Alter“ spricht, bezieht sich dies immer stärker auf die Jugend. Vor diesem Hintergrund wird die Abnahme von Fähigkeiten mit fortschreitendem Alter als inakzeptabel wahrgenommen. Das Seniorenalter, das einst mit Weisheit assoziiert wurde, gilt inzwischen als Handicap, und „Ageismus“ ist die neue Diskriminierung, die zu Sexismus und Rassismus hinzugetreten ist.

\section{Wirtschaftlicher Tod?}

In ihrem neuesten Weltbericht über Altern und Gesundheit $(2015)^{3}$ betont die WHO jedoch, dass das Seniorenalter nicht zwingend gleichbedeutend mit Abhängigkeit ist. In der Zusammenfassung des Berichts heißt es hierzu, dass „mit altersbezogenen Hypothesen zur Abhängigkeit die zahlreichen Beiträge älterer Menschen zur Wirtschaft ignoriert werden“ (2015: 8-9). Hier lässt sich die von Cook (2011) in Großbritannien durchgeführte Studie anführen, laut der Senioren für die Gesellschaft einen Nettobeitrag von etwa 40 Milliarden Pfund (£) erbringen. Das Thema ist also im Gespräch... Ist das, was am Seniorenalter unbehaglich erscheint, also nicht nur die Assoziation mit „biologischem Tod“, sondern auch mit „wirtschaftlichem Tod“? Berthoud thematisierte diese Analysegrundlagen bereits 1986 in einer Veröffentlichung. Dabei wies er darauf hin, dass das Leben ein zu managendes Kapital

\footnotetext{
${ }^{3}$ http://www.who.int/ageing/publications/world-report-2015/fr/
} 
und der Mensch zu einer Sache geworden sei. Er erinnerte daran, dass das Leben in unseren Gesellschaften nach einem von der Produktivität diktierten Rhythmus in drei Phasen verläuft: Auf die Phase, die als „Eintritt ins Erwerbsleben“ bezeichnet wird, folgt das Erwerbsleben und anschließend das Rentenalter, das gleichbedeutend ist mit dem Ausscheiden aus dem Erwerbsleben in die dritte Lebensphase, den Ruhestand. Inzwischen hat unsere Gesellschaft, in der die Lebenserwartung ansteigt, eine vierte Lebensphase erfunden. Knüpft man an die Überlegungen an, die Berthoud bereits vor über dreißig Jahren entwickelt hat, versteht man sofort, dass die aktive dritte Lebensphase in das Wirtschaftssystem einbezogen wurde und zwar nicht in Gestalt von produktiven Personen, sondern von Verbrauchern, die für die Ausweitung des Systems ebenfalls notwendig sind. Erscheint der WHOBericht in diesem Fahrwasser nicht wie ein erster Schritt, um auch die vierte Lebensphase in das kapitalistische System zu integrieren, wenn darin festgestellt wird, dass ,zahlreiche Ansatzpunkte für Maßnahmen zu finden sind, die ein Altern bei guter Gesundheit ermöglichen sollen und deren einziges Ziel es ist, die Funktionsfähigkeit zu optimieren“ (2015 : 14; Hervorhebung durch die Autoren)? Diese Optimierung hat nur Sinn in unserem westlichen Menschenbild, das auf einer bestimmten Metapher beruht: Der Körper wird dabei als Maschine aufgefasst, die instand gehalten, repariert und verbessert werden muss, um höhere Leistungen zu erzielen (Le Breton, 1999). In dem Maße, wie die Wissenschaft und Technik uns eine Leistungssteigerung ermöglichen, wird die Messlatte immer höher gelegt. Als Folge davon ist das, was wir als „,normal“ betrachten, mit Kriterien verbunden, die sich entsprechend dem technischen Fortschritt verschieben. In dieser Hinsicht ist das Beispiel der Sehkraft aufschlussreich: Seit z. B. Kurzsichtigkeit durch eine Brille oder Kontaktlinsen korrigiert werden kann, gilt es als unpassend, die Augen zusammenzukneifen, um klarer zu sehen. Es sind technische Korrekturmöglichkeiten für die Sehkraft vorhanden, also nimmt man es nicht mehr hin, weniger gut zu sehen. Die DARPA (Defense Advanced Research Projects Agency), eine Behörde des USVerteidigungsministeriums, versucht derzeit, die Sehschärfe ihrer Kampfjetpiloten zu verbessern und geht hierbei davon aus, dass die sog. normale Sehkraft in diesem Bereich nicht ausreicht. Wenn die entsprechenden Forschungen abgeschlossen sind, kann man sich daher fragen, ob Menschen, die nach den derzeitigen Maßstäben eine gute Sehkraft besitzen, in Zukunft als behindert gelten werden. Und ist es nicht auch etwas anderes, wenn man mit 20 Jahren schlecht sehen kann, als wenn die Sehkraft mit fortschreitendem Alter abnimmt? In unserer Gesellschaft wäre die Antwort wohl negativ: Beide Fälle gelten als Erkrankungen. Dies geht eindeutig zurück auf die Definition von Gesundheit, die die WHO 1946 aufgestellt und seitdem nie verändert hat: Demnach ist „Gesundheit ein Zustand umfassenden körperlichen, geistigen und sozialen Wohlbefindens und besteht nicht nur in der Abwesenheit von Krankheit und Behinderung“. Hierin ist die gesamte Utopie perfekter Gesundheit um mit Sfez (1995) zu sprechen - bereits enthalten. In einer Gesellschaft, die unter anderem durch die ständige Erzeugung neuer Verbraucherbedürfnisse geprägt ist, ist das Wohlbefinden ein relativer Zustand, der unablässig ausgeweitet werden muss. Die Entwicklung von Technologien, die der Anpassung an eine gemeinsame Norm zu einem bestimmten Zeitpunkt dienen, garantiert jedoch nicht 
die soziale Integration derjenigen, die von diesen Technologien profitieren, da sich die Maßstäbe des Wünschenswerten mit dem technologischen Fortschritt rasch ändern. Die Definition dessen, was als eine „Therapie“ gilt, weitet sich also tendenziell aus. Hinter diesem stetig wachsenden Konzept der „Therapie“ steckt die Tatsache, dass wir - extrem ausgedrückt - alle von Anfang an die gleiche Behinderung teilen: Wir sind ganz einfach Menschen mit allen damit verbundenen Einschränkungen, zu denen nicht zuletzt Altern und Tod gehören. Somit „reparieren“ wir einen von Anfang an vorhandenen Defekt!

Aimee Mullins - ein Model, der beide Beine amputiert wurden - veranschaulicht und karikiert fast, in welchem Maße die Grundgegebenheiten des Menschen als Handicap gelten: In der Tat fasst Aimee es als echten Vorteil gegenüber „,normalen“ Frauen auf, dass sie sich nicht die Beine epilieren muss, da sich an ihren Prothesen keine Haare befinden! Wenn man die obige WHO-Definition von Gesundheit zugrunde legt, könnten Haare an den Beinen dann nicht auch eines Tages als Problem im Rahmen des öffentlichen Gesundheitswesens gelten, weil sie das Wohlbefinden von Frauen beeinträchtigen? Schließlich stellt unsere Sterblichkeit aber das größte Handicap dar, gegen das wir permanent ankämpfen (Lafontaine, 2008). Und im Gesundheitssystem werden immer mehr Personen mit der Begründung behandelt, dass bei ihnen ein Risiko bestehe. So richten sich Kampagnen zur „medizinischen Vorbeugung“ an Raucher, da diese ein Risikoverhalten im Hinblick auf die Entwicklung von Krankheiten zeigen. Die ,prädiktive Medizin“ ermöglicht die Auffindung genetischer Veranlagungen, auch wiederum bei gesunden Personen. Und schließlich kümmert sich die Anti-Aging-Medizin um Falten und andere Begleiterscheinungen des fortschreitenden Alters, da wir alle zu altern und zu sterben drohen, sowohl biologisch als auch wirtschaftlich. Die amerikanische Anti-Aging-Gesellschaft ist noch neueren Datums, da sie erst 1992 gegründet wurde. Dies zeigt, dass das Altern noch nicht lange als Gesundheitsproblem gilt. Die derzeit 26.000 Mitglieder der Vereinigung verdeutlichen jedoch den Stellenwert ${ }^{4}$, den das Thema besitzt.

Es wird oft der Transhumanismus kritisiert, eine Ideologie, in der eine Strömung davon ausgeht, dass der bisherige Mensch überholt ist und sich daher Wissenschaft und Technologie zunutze machen soll, um sich selbst zu übertreffen. Dies könnte sogar zum Aussterben unserer Spezies und zur Entstehung einer höher entwickelten Spezies führen. Indem unsere Gesellschaft nun Schwäche und Tod abweist, ist sie zutiefst transhumanistisch, gesteht sich dies aber nicht ein. Wir täten also gut daran, innezuhalten und darüber nachzudenken, was uns dazu veranlasst, unsere Grenzen unentwegt ausweiten zu wollen.

\footnotetext{
${ }^{4}$ http://www.a4m.com/
} 
Auswahl aus den zitierten Quellen (Die vollständige Literatur ist bei den

Autoren zu erhalten).

Baszanger, I. (1986) Les maladies chroniques et leur ordre négocié. Revue française de sociologie, 27(1), 3-27.

Cook, J. (2011) The socio-economic contribution of older people in the UK. Work with Older People, 15(4), 141-146.

Payer, L. (1992). Disease-Mongers: How doctors, drug companies and insurers are making you feel sick. New York: Wiley .

Rossi, I. (2009) L'accompagnement en médecine. Anthropologie d'une nécessité paradoxale, Pensée plurielle 3(22), 111-122.

Sfez, L. (1995) La santé parfaite, critique d'une nouvelle utopie. Paris: Seuil.

Autorin: Dr. Daniela Cerqui ist Anthropologin und interessiert sich für das Verhältnis zwischen Technologie, Gesellschaft und insbesondere der Menschheit. Sie lehrt derzeit an der Universität Lausanne. Kontakt: Daniela.CerquiDucret@unil.ch 